\title{
BOUNDARY CROSS THEOREM IN DIMENSION 1 WITH SINGULARITIES
}

\author{
PETER PFLUG AND VIÊT-ANH NGUYÊN
}

\begin{abstract}
Let $D$ and $G$ be copies of the open unit disc in $\mathbb{C}$, let $A$ (resp. $B$ ) be a measurable subset of $\partial D$ (resp. $\partial G)$, let $W$ be the 2 -fold cross $((D \cup A) \times B) \cup$ $(A \times(B \cup G))$, and let $M$ be a relatively closed subset of $W$. Suppose in addition that $A$ and $B$ are of positive one-dimensional Lebesgue measure and that $M$ is fiberwise polar (resp. fiberwise discrete) and that $M \cap(A \times B)=\varnothing$. We determine the "envelope of holomorphy" $\widehat{W \backslash M}$ of $W \backslash M$ in the sense that any function locally bounded on $W \backslash M$, measurable on $A \times B$, and separately holomorphic on $((A \times G) \cup(D \times B)) \backslash M$ "extends" to a function holomorphic on $\widehat{W \backslash M}$.
\end{abstract}

\section{INTRODUCTION}

The first boundary cross theorem was discovered by Malgrange-Zerner in the pioneer work [23]. Subsequent results in this direction are obtained by Komatsu [15] and Drużkowski [5]. Recently, Gonchar [6, 7] has proved a more general result for the one-dimensional case. It should be noted that Airapetyan and Henkin publish a version of the edge-of-the-wedge theorem for CR manifolds (see [1] for a brief version and [2] for a complete proof). Gonchar's result could be deduced from the latter works. In the articles [18, 19, 20, 21, the authors generalize Gonchar's result to the one-dimensional case with more optimal hypotheses and to the higher dimensional case.

On the other hand, cross theorems with analytic or pluripolar singularities have been developed by many mathematicians (see, for example, [11, 12, 13, 14] and the references therein). The question naturally arises whether there exists a mixture of these two types of cross theorems, namely, a boundary cross theorem with singularities.

The purpose of this article is to establish such a theorem in a simple but very useful setting: one-dimensional case with optimal hypotheses in the spirit of our previous work [19]. This is our first step towards a general cross theorem with singularities [22] (see also [16, 17]).

Acknowledgment. The paper was started during the stay of the second author at the University of Oldenburg in 2006. He was supported by a grant from DFG, Az. PF 227/8-2. The paper was written while he was visiting the Abdus Salam

2000 Mathematics Subject Classification. Primary 32D15, 32D10.

Key words and phrases. Boundary cross theorem, fiberwise polar/discrete, holomorphic extension, harmonic measure. 
International Centre for Theoretical Physics in Trieste. He wishes to express his gratitude to these organizations.

\section{BaCkground And Statement of the MAin RESUlt}

First we introduce some notation and terminology. In this article, $E$ always denotes the open unit disc in $\mathbb{C}$. For $a \in \mathbb{C}$ and $r>0, \Delta_{a}(r)$ is the disc centered at $a$ with radius $r$. Finally, the one-dimensional Lebesgue measure is denoted by mes .

2.1. (Sub)harmonic measure. Let $\Omega \subset \mathbb{C}$ be an open set. For any function $u: \Omega \longrightarrow \mathbb{R} \cup\{-\infty\}$, let

$$
\hat{u}(z):= \begin{cases}u(z), & z \in \Omega, \\ \limsup _{\Omega \ni w \rightarrow z} u(w), & z \in \partial \Omega .\end{cases}
$$

For a set $A \subset \bar{\Omega}$ put

$$
h_{A, \Omega}:=\sup \{u: u \in \mathcal{S H}(\Omega), u \leq 1 \text { on } \Omega, \hat{u} \leq 0 \text { on } A\} \text {, }
$$

where $\mathcal{S H}(\Omega)$ denotes the cone of all functions subharmonic on $\Omega$.

The subharmonic measure of $A$ relative to $\Omega$ is the function $\omega(\cdot, A, \Omega) \in \mathcal{S H}(\Omega)$ defined by

$$
\omega(z, A, \Omega):=h_{A, \Omega}^{*}(z), \quad z \in \Omega
$$

where $h^{*}$ denotes the upper semicontinuous regularization of $h$.

If $A \subset \partial \Omega$, then $\omega(\cdot, A, \Omega)$ is also called the harmonic measure of $A$ relative to $\Omega$. In this case, $\omega(\cdot, A, \Omega)$ is a harmonic function.

We recall the following elementary property which will be used several times later on. Let $\left(A_{k}\right)_{k=1}^{\infty}$ be a sequence of measurable subsets of $\partial E$ and $A$ a measurable subset of $\partial E$ such that $\operatorname{mes}\left(A_{k}\right)>0, A_{k} \subset A_{k+1}$, and mes $\left(A \backslash \bigcup_{k=1}^{\infty} A_{k}\right)=0$. Then

$$
\omega\left(\cdot, A_{k}, E\right) \searrow \omega(\cdot, A, E) \quad \text { as } k \nearrow \infty \text {. }
$$

2.2. Angular approach regions and locally regular points. Let $D \subset \mathbb{C}$ be a Jordan domain. Fix a conformal mapping $\Phi$ from $D$ onto $E$ which extends continuously from $\bar{D}$ onto $\bar{E}$. For $\zeta \in \partial D$ and $0<\alpha<\frac{\pi}{2}$, the Stolz region or angular approach region $\mathcal{A}_{\alpha}(\zeta)$ is given by

$$
\mathcal{A}_{\alpha}(\zeta):=\left\{\Phi^{-1}(t): t \in E \text { and }\left|\arg \left(\frac{\Phi(\zeta)-t}{\Phi(\zeta)}\right)\right|<\alpha\right\}
$$

where arg: $\mathbb{C} \longrightarrow(-\pi, \pi]$ is as usual the argument function.

Let $A \subset \bar{D}$. We say that a point $\zeta \in \bar{D}$ is a locally regular point relative to $A$ if

$$
\lim _{D \cap \Delta_{\zeta}(r) \ni z \rightarrow \zeta} \omega\left(z, A \cap \Delta_{\zeta}(r), D \cap \Delta_{\zeta}(r)\right)=0, \quad r>0 .
$$

Obviously, $\zeta \in \bar{A}$. The set of all locally regular points relative to $A$ is denoted by $A^{*}$. $A$ is said to be locally regular if $A \subset A^{*}$. 
If $A \subset \partial D$ is measurable, then it is classical that $\Phi\left(A^{*}\right)$ contains all density-points of $\Phi(A)$, hence mes $\left(\Phi\left(A \backslash\left(A \cap A^{*}\right)\right)\right)=0$, and $A \cap A^{*}$ is again locally regular. Moreover, it follows from (2.1) that

$$
\omega\left(\cdot, A \cap A^{*}, D\right)=\omega(\cdot, A, D) .
$$

Recall from Definition 4.8 in [19] the following definition. A point $\zeta \in \partial D$ is said to be an end-point of an open subset $\Omega \subset D$ if, for every $0<\alpha<\frac{\pi}{2}$, there is an open neighborhood $U=U_{\alpha}$ of $\zeta$ such that $U \cap \mathcal{A}_{\alpha}(\zeta) \subset \Omega$. The set of all end-points of $\Omega$ is denoted by $\operatorname{End}(\Omega)$.

We say that a function $f$, defined in an open subset $\Omega \subset D$, admits an angular limit $\lambda \in \mathbb{C}$ at a point $a \in \operatorname{End}(\Omega)$ if

$$
\lim _{\mathcal{A}_{\alpha}(a) \cap \Omega \ni z \rightarrow a} f(z)=\lambda, \quad 0<\alpha<\frac{\pi}{2} .
$$

2.3. Cross and separate holomorphy. Let $D, G \subset \mathbb{C}$ be two open sets, let $A$ (resp. $B$ ) be a subset of $\bar{D}$ (resp. $\bar{G}$ ). We define a 2 -fold cross $W$, its interior $W^{\mathrm{o}}$ as

$$
\begin{aligned}
W & :=\mathbb{X}(A, B ; D, G):=((D \cup A) \times B) \cup(A \times(B \cup G)), \\
W^{\mathrm{o}} & :=\mathbb{X}^{\mathrm{o}}(A, B ; D, G):=(D \times B) \cup(A \times G) .
\end{aligned}
$$

For a 2 -fold cross $W:=\mathbb{X}(A, B ; D, G)$ define

$$
\widehat{W}=\widehat{\mathbb{X}}(A, B ; D, G):=\{(z, w) \in D \times G: \omega(z, A, D)+\omega(w, B, G)<1\} .
$$

Let $M$ be a subset of $W$. Then the fibers $M_{a}$ and $M^{b}$ are given by

$$
M_{a}:=\{w \in G:(a, w) \in M\} \quad(a \in A) ; \quad M^{b}:=\{z \in D:(z, b) \in M\} \quad(b \in B) .
$$

We say that $M$ possesses a certain property in fibers over $A$ (resp. over $B$ ) if all fibers $M_{a}$ with $a \in A$ (resp. all fibers $M^{b}$ with $b \in B$ ) possess this property.

Suppose that $M$ is relatively closed in fibers over $A$ and $B$. We say that a function $f: W \backslash M \longrightarrow \mathbb{C}$ is separately holomorphic on $W^{o} \backslash M$ and write $f \in \mathcal{O}_{s}\left(W^{\mathrm{o}} \backslash M\right)$, if for any $a \in A$ (resp. $b \in B$ ) the function $\left.f(a, \cdot)\right|_{G \backslash M_{a}}$ (resp. $\left.f(\cdot, b)\right|_{D \backslash M^{b}}$ ) is holomorphic.

From now on we assume, in addition, that $D$ and $G$ are Jordan domains, and $A \subset \partial D, B \subset \partial G$. Then we define the regular part $W^{*}$ relative to $W$ as

$$
W^{*}:=\mathbb{X}\left(A^{*}, B^{*} ; D, G\right) .
$$

Let $\Omega$ be an open subset of $D \times G$. A point $(a, b) \in A^{*} \times G$ (resp. $\left.(a, b) \in D \times B^{*}\right)$ is said to be an end-point of $\Omega$ if, for every $0<\alpha<\frac{\pi}{2}$, there are an open neighborhood $U=U_{\alpha}$ of $a$ and an open neighborhood $V=V_{\alpha}$ of $b$ such that

$$
\left(U \cap \mathcal{A}_{\alpha}(a)\right) \times V \subset \Omega \quad\left(\text { resp. } U \times\left(V \cap \mathcal{A}_{\alpha}(b)\right) \subset \Omega\right) .
$$

The set of all end-points of $\Omega$ is denoted by $\operatorname{End}(\Omega)$. We say that a function $f$ : $\Omega \longrightarrow \mathbb{C}$ admits an angular limit $\lambda \in \mathbb{C}$ at $(a, b) \in \operatorname{End}(\Omega)$ if under the previous 
notation one of the following cases occurs:

Case 1: $(a, b) \in A^{*} \times G$ and the following limits exist and are equal to $\lambda$

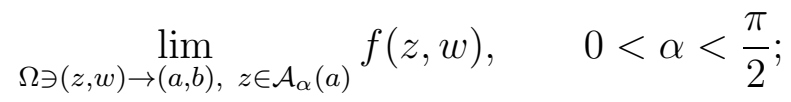

Case 2: $(a, b) \in D \times B^{*}$ and the following limits exist and are equal to $\lambda$

$$
\lim _{\Omega \ni(z, w) \rightarrow(a, b),} f(z, w), \quad 0<\alpha<\frac{\pi}{2} .
$$

For an open set $\Omega \subset \mathbb{C}^{k}$, let $\mathcal{O}(\Omega)$ denote the space of all holomorphic functions on $\Omega$. A function $f: \mathcal{P} \rightarrow \mathbb{C}$, where $\mathcal{P}$ is a topological space, is said to be locally bounded, if for every point $p \in \mathcal{P}$ there exists a neighborhood $U$ of $p$ such that $\sup _{U}|f|<\infty$.

2.4. Statement of the main result. Now we are able to state the following

Main Theorem. Let $D=G=E$ and let $A \subset \partial D, B \subset \partial G$ be measurable subsets such that $\operatorname{mes}(A)>0, \operatorname{mes}(B)>0$. Consider the cross $W:=\mathbb{X}(A, B ; D, G)$. Let $M$ be a relatively closed subset of $W$ such that

- $M_{a}$ is polar (resp. discrete) in $G$ for all $a \in A$ and $M^{b}$ is polar (resp. discrete) in $D$ for all $b \in B$

- $M \cap(A \times B)=\varnothing$.

Then there exists a relatively closed pluripolar subset (resp. an analytic subset) $\widehat{M}$ of $\widehat{W}$ with the following two properties:

(i) The set of end-points of $\widehat{W} \backslash \widehat{M}$ contains $\left(W^{o} \cap W^{*}\right) \backslash M$.

(ii) Let $f: W \backslash M \longrightarrow \mathbb{C}$ be a locally bounded function such that

- for all $a \in A,\left.f(a, \cdot)\right|_{G \backslash M_{a}}$ is holomorphic and admits the angular limit $f(a, b)$ at all points $b \in B$;

- for all $b \in B,\left.f(\cdot, b)\right|_{D \backslash M^{b}}$ is holomorphic and admits the angular limit $f(a, b)$ at all points $a \in A$;

- $\left.f\right|_{A \times B}$ is measurable.

Then there is a unique function $\hat{f} \in \mathcal{O}(\widehat{W} \backslash \widehat{M})$ such that $\hat{f}$ admits the angular limit $f$ at all points of $\left(W^{o} \cap W^{*}\right) \backslash M$.

Moreover, if $M=\varnothing$, then $\widehat{M}=\varnothing$.

\section{Preparatory Results}

3.1. Auxiliary results. First recall the following well-known result (see, for example, [10]).

Theorem 3.1. Let $D, G$ and $A, B$ be open subsets of $\mathbb{C}$ such that $A \subset D$ and $B \subset G$. Put $W:=\mathbb{X}(A, B ; D, G)$ and $\widehat{W}:=\widehat{\mathbb{X}}(A, B ; D, G)$. Then $W \subset \widehat{W}$ and every function $f \in \mathcal{O}_{s}(W)$ extends uniquely to a function $\hat{f} \in \mathcal{O}(\widehat{W})$.

\footnotetext{
${ }^{1}$ In other words, $M$ is polar (resp. discrete) in fibers over $A$ and $B$.
} 
The following mixed cross theorem has been proved in [19, Theorem 7.3] (see also [17. Theorem 4.2] for another proof using the method of holomorphic discs).

Theorem 3.2. Let $A$ be a measurable subset of $\partial E$ such that $A$ is locally regular. Let $G \subset \mathbb{C}$ be an open set and $B$ an open subset of $G$. For $0 \leq \delta<1$ put $\Omega:=$ $\{z \in E: \omega(z, A, E)<1-\delta\}$. Let $W:=\mathbb{X}(A, B ; \Omega, G), W^{o}:=\mathbb{X}^{o}(A, B ; \Omega, G)$, and

$$
\widehat{\widetilde{W}}=\widehat{\widetilde{\mathbb{X}}}(A, B ; \Omega, G):=\left\{(z, w) \in E \times G: \frac{\omega(z, A, E)}{1-\delta}+\omega(w, B, G)<1\right\} .
$$

Let $f: W \longrightarrow \mathbb{C}$ be such that

(i) $f \in \mathcal{O}_{s}\left(W^{o}, \mathbb{C}\right)$;

(ii) $f$ is locally bounded on $W,\left.f\right|_{A \times B}$ is a measurable function;

(iii) for all $w \in B, 3$

$$
\lim _{\mathcal{A}_{\alpha}(a) \ni z \rightarrow a} f(z, w)=f(a, w), \quad a \in A, 0<\alpha<\frac{\pi}{2} .
$$

Then there exists a unique function $\hat{f} \in \mathcal{O}(\widehat{\widetilde{W}})$ such that $\hat{f}=f$ on $\Omega \times B$ and

$$
\lim _{\mathcal{A}_{\alpha}(a) \ni z \rightarrow a} \hat{f}(z, w)=f(a, w), \quad a \in A, w \in G, 0<\alpha<\frac{\pi}{2} .
$$

Moreover, $|f|_{W}=|\hat{f}|_{\widehat{W}}$.

The next result proved by the authors in [19] generalizes the work of Gonchar in [6. 7].

Theorem 3.3. We keep the hypotheses and notation of the Main Theorem. Suppose in addition that $M=\varnothing$. Then the conclusion of the Main Theorem holds for $\widehat{M}=\varnothing$.

The following two extension theorems are also needed in the sequel.

Theorem 3.4 (Chirka [4]). Let $D \subset \mathbb{C}^{n}$ be a domain and let $\widehat{D}$ be the envelope of holomorphy of D. Assume that $S$ is a relatively closed pluripolar subset of $D$. Then there exists a relatively closed pluripolar subset $\widehat{S}$ of $\widehat{D}$ such that $\widehat{S} \cap D \subset S$ and $\widehat{D} \backslash \widehat{S}$ is the envelope of holomorphy of $D \backslash S$.

Theorem 3.5 (Imomkulov-Khujamov 8, Imomkulov [9]). Let $A$ be a measurable subset of $\partial E$ with $\operatorname{mes}(A)>0$, let $M$ be a relatively closed subset of $A \times(\mathbb{C} \backslash \bar{E})$ such that $M_{a}:=\{w \in \mathbb{C}:(a, w) \in M\}$ is polar (resp. finite) for all $a \in A$. Then there exists a relatively closed pluripolar (resp. analytic) subset $S$ of $E \times(\mathbb{C} \backslash \bar{E})$ with the following property:

Let $f:(E \cup A) \times E \longrightarrow \mathbb{C}$ be bounded, $\left.f\right|_{E \times E} \in \mathcal{O}(E \times E)$ such that $\lim _{z \in \mathcal{A}_{\alpha}(a)} f(z, w)=f(a, w)$ for all $a \in A, w \in E$ and $0<\alpha<\frac{\pi}{2}$. Moreover, assume that the (holomorphic) function $f(a, \cdot)$ extends to a holomorphic function on $\mathbb{C} \backslash M_{a}$ for every $a \in A$. Then $\left.f\right|_{E \times E}$ extends holomorphically to $(E \times \mathbb{C}) \backslash S$.

\footnotetext{
${ }^{2}$ It will be shown in Lemma 3.6 below that $\widetilde{\omega}(\cdot, A, \Omega)=\frac{\omega(\cdot, A, E)}{1-\delta}$ on $\Omega$, where $\widetilde{\omega}(\cdot, A, \Omega)$ is, in some sense, the "angular" version of the harmonic measure.

${ }^{3}$ Since $A$ is locally regular, it follows that $A \subset \operatorname{End}(\Omega)$.

${ }^{4}$ Since $A$ is locally regular, we have $A \times G \subset \operatorname{End}(\widehat{\widehat{W}})$.
} 
Proof. This is a slightly modified version of the result in [8]. In fact, ImomkulovKhujamov suppose that $\left.f\right|_{E \times E}$ can be extended continuously onto $\bar{E} \times \bar{E}$. But their proof still works under the hypotheses of Theorem 3.5. Consequently, for each function $f$ as in the statement of the theorem, there is a relatively closed pluripolar (resp. analytic) subset $S_{f}$ of $E \times(\mathbb{C} \backslash \bar{E})$ such that $f$ extends to a holomorphic function on $G_{f}:=(E \times \mathbb{C}) \backslash S_{f}$ and that the latter function does not extend holomorphically across any point of $S_{f}$. Let $G$ denote the connected component of the interior of $\bigcap_{f} G_{f}$ that contains $E^{2}$ and let $S:=(E \times \mathbb{C}) \backslash G$. It remains to show that $S$ is pluripolar (resp. analytic).

Take $(a, b) \in\left(\left(A \cap A^{*}\right) \times \mathbb{C}\right) \backslash M$. Since $M$ is relatively closed in $A \times \mathbb{C}$ and $M_{a}$ is polar, there exists a smooth curve $\gamma:[0,1] \rightarrow \mathbb{C} \backslash M_{a}$ such that $\gamma(0)=0, \gamma(1)=b$. Take an $\epsilon>0$ so small that

$$
\left(\Delta_{a}(\epsilon) \times\left(\gamma([0,1])+\Delta_{0}(\epsilon)\right)\right) \cap M=\varnothing
$$

and that $V_{b}:=\Delta_{0}\left(\frac{1}{2}\right) \cup\left(\gamma([0,1])+\Delta_{0}(\epsilon)\right)$ is a Jordan domain. Consider the cross

$$
Y:=\mathbb{X}\left(A \cap \Delta_{a}(\epsilon), \partial V_{b} \cap \partial \Delta_{0}\left(\frac{1}{2}\right) ; \Delta_{a}(\epsilon) \cap E, V_{b}\right) .
$$

Then $\left.f\right|_{Y}$ satisfies the hypotheses of Theorem 3.3. Consequently, we get $\widehat{Y} \subset G_{f}$ for all $f$ as in the statement of the theorem. Hence $\widehat{Y} \subset G$. Thus $S_{a}^{*} \subset M_{a}$ for all $a \in A$, where $S_{a}^{*}$ is the non-tangential boundary layer of a pseudoconcave set $S$ (see [9, p. 358]). Consequently, by Lemma 6 and 7 from [9] (see also Lemma 7 and 8 in [8]), $S$ is pluripolar (resp. analytic).

3.2. Two techniques and their applications. The technique level sets of (plurisub)harmonic measure was introduced by the authors in [18]. However, it turns out that it can be successfully used in solving many problems arising from the theory of separately holomorphic and meromorphic mappings (see [19, 20, 16, 17]). For an open set $D \subset \mathbb{C}$, a subset $A \subset \partial D$, and $0<\delta<1$ the $\delta$-level set of the harmonic measure $\omega(\cdot, A, D)$ is, by definition,

$$
D_{A, \delta}:=\{z \in D: \omega(z, A, D)<\delta\} .
$$

The technique of level sets consists in "replacing" $A$ (resp. $D)$ by $D_{A, \delta}\left(\operatorname{resp} . D_{A, 1-\delta}\right)$ for a suitable $0<\delta<\frac{1}{2}$.

Recall the following property of the level sets.

Lemma 3.6. Let $D$ be either an empty set or a Jordan domain such that $E \not \subset D$ and that $D \cup E$ is a Jordan domain. For a measurable subset $A$ of $\partial E \cap \partial(D \cup E)$ with $\operatorname{mes}(A)>0$ and $0<\delta<1$ let $\Omega_{\delta}:=E_{A, \delta} \cup D$. Define the angular harmonic measure

$$
\widetilde{\omega}\left(z, A, \Omega_{\delta}\right):=\sup _{u \in \mathcal{U}_{A, \delta}} u(z), \quad z \in \Omega_{\delta}
$$

where $\mathcal{U}_{A, \delta}$ is the cone of all subharmonic functions $u \leq 1$ on $\Omega_{\delta}$ such that

$$
\limsup _{\Omega_{\delta} \cap \mathcal{A}_{\alpha}(\zeta) \ni z \rightarrow \zeta} u(z) \leq 0, \quad \zeta \in A \cap A^{*}, 0<\alpha<\frac{\pi}{2} .
$$


1) If $D=\varnothing$, then $\widetilde{\omega}\left(z, A, \Omega_{\delta}\right)=\frac{\omega(z, A, E)}{1-\delta}, z \in \Omega_{\delta}$.

2) If $D$ is a Jordan domain, then $\widetilde{\omega}\left(z, A, \Omega_{\delta}\right) \searrow \omega(z, A, E \cup D)$ as $\delta \searrow 0^{+}$.

Proof. Part 1) follows from [19, Theorem 4.10]. Part 2) is a consequence of Part 1).

The technique conformal mappings has been introduced by the second author in [17. This allows to reduce the study of holomorphic extensions on some level sets to the unit disc.

The main idea of the technique of conformal mappings is described below (see Proposition 5.2 in [17] for a proof).

Proposition 3.7. Let $A$ be a measurable subset of $\partial E$ with mes $(A)>0$. For $0 \leq$ $\delta<1$ put $G:=\{w \in E: \omega(w, A, E)<1-\delta\}$. Let $\Omega$ be an arbitrary connected component of $G$. Then

$1) \operatorname{End}(\Omega)$ is a measurable subset of $\partial E$ and $\operatorname{mes}(\operatorname{End}(\Omega))>0$. Moreover, $\Omega$ is a simply connected domain.

In virtue of Part 1) and the Riemann mapping theorem, let $\Phi$ be a conformal mapping of $\Omega$ onto $E$.

2) For every $\zeta \in \operatorname{End}(\Omega)$, there is $\eta \in \partial E$ such that

$$
\lim _{\Omega \cap \mathcal{A}_{\alpha}(\zeta) \ni z \rightarrow \zeta} \Phi(z)=\eta, \quad 0<\alpha<\frac{\pi}{2} .
$$

$\eta$ is called the limit of $\Phi$ at the end-point $\zeta$ and it is denoted by $\Phi(\zeta)$. Moreover, $\left.\Phi\right|_{\operatorname{End}(\Omega)}$ is one-to-one.

3) Let $f$ be a bounded holomorphic function on $\Omega, \zeta \in \operatorname{End}(\Omega)$, and $\lambda \in \mathbb{C}$ such that $\lim _{\Omega \cap \mathcal{A}_{\alpha}(\zeta) \ni z \rightarrow \zeta} f(z)=\lambda$ for some $0<\alpha<\frac{\pi}{2}$. Then $f \circ \Phi^{-1} \in \mathcal{O}(E)$ admits the angular limit $\lambda$ at $\Phi(\zeta)$.

4) Let $\Delta$ be a measurable subset of $\operatorname{End}(\Omega)$ such that $\operatorname{mes}(\Delta)=\operatorname{mes}(\operatorname{End}(\Omega))$. Put $\Phi(\Delta):=\{\Phi(\zeta), \zeta \in \Delta\}$, where $\Phi(\zeta)$ is given by Part 2). Then $\Phi(\Delta)$ is a measurable subset of $\partial E$ with mes $(\Phi(\Delta))>0$ and

$$
\omega(\Phi(z), \Phi(\Delta), E)=\frac{\omega(z, A, E)}{1-\delta}, \quad z \in \Omega .
$$

As an application of the technique of conformal mappings, we give the following extended version of Theorem 3.5.

Theorem 3.8. Let $A$ be a measurable subset of $\partial E$ with $\operatorname{mes}(A)>0$. For a given $0 \leq \delta<1$ put $\Omega:=\{w \in E: \omega(w, A, E)<1-\delta\}$. Let

$$
f:(\Omega \cup(A \cap \operatorname{End}(\Omega))) \times E \longrightarrow \mathbb{C}
$$

be a bounded function such that $\left.f\right|_{\Omega \times E}$ is holomorphic and $\lim _{z \rightarrow a, z \in \mathcal{A}_{\alpha}(a)} f(z, w)=$ $f(a, w)$ for all $a \in A \cap \operatorname{End}(\Omega), w \in E$ and $0<\alpha<\frac{\pi}{2}$. Suppose in addition that for every $a \in A \cap \operatorname{End}(\Omega)$, the function $f(a, \cdot)$ is holomorphic and it extends to $a$ holomorphic function on the whole plane except for a closed polar (resp. finite) set of singularities. Then $\left.f\right|_{\Omega \times E}$ extends holomorphically to $(\Omega \times \mathbb{C}) \backslash S$, where $S$ is a relatively closed pluripolar (resp. analytic) subset of $\Omega \times \mathbb{C}$. 
Theorem 3.5 is a special case of the above result for $\delta=0$.

Proof. We only treat the case where the set of singularities of $f(a, \cdot)$ is closed polar for $a \in A \cap \operatorname{End}(\Omega)$. Since the remaining case where these sets are finite is analogous, it is therefore left to the interested reader. Using (2.2) we may suppose without loss of generality that $A$ is locally regular. Then $A \subset \operatorname{End}(\Omega)$. Let $\left(\Omega_{k}\right)_{k \in K}$ be the family of all connected components of $\Omega$, where $K$ is a countable index set. By Theorem 4.9 in [19],

$$
\begin{aligned}
\operatorname{End}(\Omega)= & \bigcup_{k \in K} \operatorname{End}\left(\Omega_{k}\right), \quad \operatorname{mes}\left(\operatorname{End}\left(\Omega_{k}\right) \cap A\right)=\operatorname{mes}\left(\operatorname{End}\left(\Omega_{k}\right)\right), \\
& \operatorname{End}\left(\Omega_{k}\right) \cap \operatorname{End}\left(\Omega_{k^{\prime}}\right)=\varnothing \quad \text { for } k \neq k^{\prime} .
\end{aligned}
$$

By Proposition 3.7, we may fix a conformal mapping $\Phi_{k}$ from $\Omega_{k}$ onto $E$ for every $k \in K$. Put

$$
A_{k}:=\Phi_{k}\left(\operatorname{End}\left(\Omega_{k}\right) \cap A\right), W_{k}:=\left(E \cup A_{k}\right) \times E, \quad k \in K .
$$

Recall from the hypothesis that for every fixed $w \in E$, the holomorphic function $\left.f(\cdot, w)\right|_{\Omega}$ is bounded and that for every $\zeta \in A \cap \operatorname{End}(\Omega)$,

$$
\lim _{\Omega \cap \mathcal{A}_{\alpha}(\zeta) \ni z \rightarrow \zeta} f(z, w)=f(\zeta, w), \quad 0<\alpha<\frac{\pi}{2} .
$$

Consequently, Part 3) of Proposition 3.7, applied to $\left.f(\cdot, w)\right|_{\Omega_{k}}$ with $k \in K$, implies that for every fixed $w \in E, f\left(\Phi_{k}^{-1}(\cdot), w\right) \in \mathcal{O}(E)$ admits the angular limit $f(\zeta, w)$ at $\Phi_{k}(\zeta)$ for all $\zeta \in A \cap \operatorname{End}\left(\Omega_{k}\right)$. By Part 1$)$ of that proposition, we know that mes $(A \cap$ $\left.\operatorname{End}\left(\Omega_{k}\right)\right)>0$. This discussion and the hypothesis allow us to apply Theorem 3.5 to the function $g_{k}: W_{k} \longrightarrow \mathbb{C}$ defined by

$$
g_{k}(z, w):=\left\{\begin{array}{ll}
f\left(\Phi_{k}^{-1}(z), w\right), & (z, w) \in E \times E \\
f\left(\Phi_{k}^{-1}(z), w\right), & (z, w) \in A_{k} \times E
\end{array},\right.
$$

where in the second line we have used the definition of $\left.\Phi_{k}\right|_{\operatorname{End}\left(\Omega_{k}\right)}$ and its one-to-one property proved by Part 2) of Proposition 3.7. Consequently, we obtain a relatively closed pluripolar set $S_{k} \subset E \times \mathbb{C}$ such that $S_{k} \cap(E \times E)=\varnothing$ and that $\left.g_{k}\right|_{E \times E}$ extends holomorphically to a function $\hat{g}_{k} \in \mathcal{O}\left((E \times \mathbb{C}) \backslash S_{k}\right)$ with

$$
\lim _{\mathcal{A}_{\alpha}(a) \ni z \rightarrow a} \hat{g}_{k}(z, w)=g_{k}(a, w), \quad(a, w) \in A_{k} \times E .
$$

Put

$$
\widehat{\mathcal{W}}_{k}:=\left\{\left(\Phi_{k}^{-1}(z), w\right),(z, w) \in(E \times \mathbb{C}) \backslash S_{k}\right\}, \quad k \in K .
$$

Observe that the open sets $\left(\widehat{\mathcal{W}}_{k}\right)_{k \in K}$ are pairwise disjoint. Moreover, by (3.1),

$$
\begin{aligned}
\bigcup_{k \in K} \widehat{\mathcal{W}}_{k}= & \bigcup_{k \in K}\left\{(z, w) \in \Omega_{k} \times \mathbb{C}:\left(\Phi_{k}(z), w\right) \notin S_{k}\right\} \\
& =(\Omega \times \mathbb{C}) \backslash \bigcup_{k \in K}\left\{(z, w) \in \Omega_{k} \times \mathbb{C}:\left(\Phi_{k}(z), w\right) \in S_{k}\right\}=:(\Omega \times \mathbb{C}) \backslash S .
\end{aligned}
$$


Since $S_{k}$ is relatively closed pluripolar in $E \times \mathbb{C}$ for $k \in K$, we see that $S$ is relatively closed pluripolar in $\Omega \times \mathbb{C}$. Therefore, we define the desired extension function $\hat{f} \in$ $\mathcal{O}((\Omega \times \mathbb{C}) \backslash S)$ by the formula

$$
\hat{f}(z, w):=\hat{g}_{k}\left(\Phi_{k}(z), w\right), \quad(z, w) \in \Omega_{k}, k \in K .
$$

This, combined with (3.1)-(3.3), implies that $\lim _{\mathcal{A}_{\alpha}(a) \ni z \rightarrow a} \hat{f}(z, w)=f(a, w)$ for all $(a, w) \in A \times E$. The uniqueness of $\hat{f}$ follows from the one of $\hat{g}_{k}, k \in K$. Hence, the proof of the theorem is complete.

3.3. Gluing theorems. The following theorems will be very useful in the next sections when we need to glue different local extensions.

Theorem 3.9. Let $A$ and $\mathcal{N}$ be measurable subsets of $\partial E$ with $\operatorname{mes}(\mathcal{N})=0$. Let $0<\delta<1$ and $E_{A, \delta}:=\{z \in E: \omega(z, A, E)<\delta\}$. Suppose that $f \in \mathcal{O}\left(E_{A, \delta}\right)$ admits the angular limit 0 at all points of $\left(A \cap A^{*}\right) \backslash \mathcal{N}$. Then $f \equiv 0$.

Proof. See Theorem 5.4 in [19].

Theorem 3.10. Let $(\Omega)_{i \in I}$ be a family of open subsets of an open set $\Omega \subset \mathbb{C}^{n}$. Let $M$ a relatively closed pluripolar subset (resp. an analytic subset) of $\Omega$ and $M_{i}$ a non pluripolar subset of $\Omega_{i}$ such that $M \cap M_{i}=\varnothing, i \in I$. Suppose that $f \in \mathcal{O}(\Omega \backslash M)$ and $f_{i} \in \mathcal{O}\left(\Omega_{i}\right)$ satisfy $f=f_{i}$ on $M_{i}$ for $i \in I$. Then there exist a relatively closed pluripolar subset (resp. an analytic subset) $\widehat{M} \subset \Omega$ and a function $\hat{f} \in \mathcal{O}(\Omega \backslash \widehat{M})$ such that $\widehat{M} \subset M$ and $\hat{f}=f$ on $\Omega \backslash M$, and that for all $i \in I$, we have $\widehat{M} \cap \Omega_{i}=\varnothing$ and $\hat{f}=f_{i}$ on $\Omega_{i}$.

Proof. The case where $M$ is a relatively closed pluripolar subset of $\Omega$ is not difficult. The remaining case where $M$ is an analytic subset of $\Omega$ follows from an easy application of Proposition 3.4.5 in [10].

Theorem 3.11. Let $\left(\Omega_{n}\right)_{n=1}^{\infty}$ be an increasing sequence of open subsets of an open set $\Omega \subset \mathbb{C}^{n}$ such that $\Omega_{n} \nearrow \Omega$ as $n \nearrow \infty$. For every $n \in \mathbb{N}$ let $M_{n}$ be a relatively closed pluripolar subset (resp. an analytic subset) of $\Omega_{n}$ and $f_{n} \in \mathcal{O}\left(\Omega_{n} \backslash M_{n}\right)$. Suppose in addition that $f_{n}=f_{n+1}$ on $\Omega_{n} \backslash\left(M_{n} \cup M_{n+1}\right), n \in \mathbb{N}$. Then there exist a relatively closed pluripolar subset (resp. an analytic subset) $M \subset \Omega$ and a function $f \in \mathcal{O}(\Omega \backslash M)$ such that $M \cap \Omega_{n} \subset M_{n}$ and $f=f_{n}$ on $\Omega_{n} \backslash M_{n}$ for all $n \in \mathbb{N}$.

Proof. It is left to the interested reader.

\section{Extensions through the SINGUlarities}

We keep the hypotheses and notation of the Main Theorem. Moreover, we only give the proof for the case where the singular set is fiberwise polar, that is, $M_{a}$ (resp. $M^{b}$ ) is polar in $G$ (resp. $D$ ) for all $a \in A$ (resp. $b \in B$ ). Since the remaining case where the singular set is fiberwise discrete is analogous, it is therefore left to the interested reader.

In this section and the beginning of the next one we assume that

$A$ and $B$ are compact sets. 
This assumption will be removed at the end of the next section.

Since $(A \times B) \cap M=\varnothing$ (by the hypothesis), we may find $N$ points $a_{1}, \ldots, a_{N} \in A$, $N$ numbers $r_{1}, \ldots, r_{N}>0, N^{\prime}$ points $b_{1}, \ldots, b_{N^{\prime}} \in B$, and $N^{\prime}$ numbers $s_{1}, \ldots, s_{N^{\prime}}>$ 0 such that

$$
A \subset \bigcup_{k=1}^{N} \Delta_{a_{k}}\left(r_{k}\right), \quad B \subset \bigcup_{l=1}^{N^{\prime}} \Delta_{b_{l}}\left(s_{l}\right), \quad M \cap\left(\bigcup_{k=1}^{N} \Delta_{a_{k}}\left(r_{k}\right) \times \bigcup_{l=1}^{N^{\prime}} \Delta_{b_{l}}\left(s_{l}\right)\right)=\varnothing
$$

Put

$$
\widetilde{D}:=D \cap \bigcup_{k=1}^{N} \Delta_{a_{k}}\left(r_{k}\right), \quad \widetilde{G}:=G \cap \bigcup_{l=1}^{N^{\prime}} \Delta_{b_{l}}\left(s_{l}\right) .
$$

Then it is clear that $\mathbb{X}(A, B ; \widetilde{D}, \widetilde{G}) \cap M=\varnothing$.

We introduce the following notation. For an $a \in A($ resp. $b \in B$ ) and $0<r, \delta<1$, let

$$
\begin{aligned}
D_{a, r, \delta} & :=\left\{z \in D \cap \Delta_{a}(r): \omega\left(z, A \cap \Delta_{a}(r), D \cap \Delta_{a}(r)\right)<\delta\right\}, \\
G_{b, r, \delta} & :=\left\{w \in G \cap \Delta_{b}(r): \omega\left(w, B \cap \Delta_{b}(r), G \cap \Delta_{b}(r)\right)<\delta\right\} .
\end{aligned}
$$

Let $\Omega$ be an open subset of $\widehat{W}$. A point $(a, b) \in\left(A \cap A^{*}\right) \times G$ (resp. $(a, b) \in$ $\left.D \times\left(B \cap B^{*}\right)\right)$ is said to be a strong end-point of $\Omega$ if there exist $0<r, \delta<1$ and an open neighborhood $V$ of $b$ (resp. and an open neighborhood $U$ of $a$ ) such that

$$
D_{a, r, \delta} \times V \subset \Omega \quad\left(\text { resp. } U \times G_{b, r, \delta} \subset \Omega\right) .
$$

It is clear that a strong end-point of $\Omega$ is also an end-point. But the converse statement is in general false.

Now, we are in the position to extend $f$ holomorphically through the singular set $M$.

Proposition 4.1. For any $a \in A \cap A^{*}, w \in G$, there exist $r, \rho, \delta \in(0,1)$ and $a$ relatively closed pluripolar subset $S \subset D_{a, r, \delta} \times \Delta_{w}(\rho)$ with the following properties:

1) $\Delta_{w}(\rho) \subset G$ and the set

$$
T:=\left(\left(A \cap A^{*} \cap \Delta_{a}(r)\right) \times \Delta_{w}(\rho)\right) \backslash M
$$

is contained in the set of strong end-points of $\left(D_{a, r, \delta} \times \Delta_{w}(\rho)\right) \backslash S$.

2) There is a function $\hat{f} \in \mathcal{O}\left(\left(D_{a, r, \delta} \times \Delta_{w}(\rho)\right) \backslash S\right)$ which admits the angular limit $f$ at all points of $T$.

Proof. Fix an $a_{0} \in A \cap A^{*}$ and a $w_{0} \in G$ as in the proposition. First we determine $0<r, \rho, \delta<1$ and then we will construct a function $\hat{f} \in \mathcal{O}\left(\left(D_{a_{0}, r, \delta} \times \Delta_{w_{0}}(\rho)\right) \backslash \widetilde{S}\right)$, where $\widetilde{S}$ is a relatively closed pluripolar subset of $D_{a_{0}, r, \delta} \times \Delta_{w_{0}}(\rho)$.

Since $M_{a_{0}}$ is a relatively closed polar set in $G$, one may choose $\rho>0$ such that $\Delta_{w_{0}}(\rho) \Subset G$ and $M_{a_{0}} \cap \partial \Delta_{w_{0}}(\rho)=\varnothing$ (cf. [3], Theorem 7.3.9). Take $\rho^{-}, \rho^{+}>0$ such that $\rho^{-}<\rho<\rho^{+}, \Delta_{w_{0}}\left(\rho^{+}\right) \Subset G$, and $M_{a_{0}} \cap \bar{P}=\varnothing$, where

$$
P:=\left\{w \in \mathbb{C}: \rho^{-}<\left|w-w_{0}\right|<\rho^{+}\right\} .
$$


Define

$$
\mathcal{G}:=\left\{w \in \widetilde{G}: \omega(w, B, \widetilde{G})<\frac{1}{2}\right\} .
$$

Let $\gamma:[0,1] \rightarrow G \backslash M_{a_{0}}$ be a curve such that $\gamma(0) \in \mathcal{G}, \gamma(1) \in \partial \Delta_{w_{0}}(\rho)$. Since $M$ is relatively closed in $W$, there exist $r, t \in(0,1)$ such that

$$
\Delta_{a_{0}}(r) \cap D \subset \widetilde{D} \quad \text { and } \quad\left(A \cap \Delta_{a_{0}}(r)\right) \times\left(\left(\gamma([0,1])+\Delta_{0}(t)\right) \cup P\right) \subset W \backslash M
$$

Put

$$
V:=\mathcal{G} \cup\left(\gamma([0,1])+\Delta_{0}(t)\right) \cup P
$$

and consider the cross

$$
Y:=\mathbb{X}\left(A \cap A^{*} \cap \Delta_{a_{0}}(r), \mathcal{G} ; D_{a_{0}, r, \frac{1}{2}}, V\right) .
$$

Using (4.1) and (4.3) and the hypotheses on $f$ in the Main Theorem, we are able to apply Theorem 3.3 to the function $f$ restricted to $\mathbb{X}\left(A \cap \Delta_{a_{0}}(r), B ; D \cap \Delta_{a_{0}}(r), \widetilde{G}\right)$. Consequently, we obtain $\tilde{f} \in \mathcal{O}\left(\widehat{\mathbb{X}}\left(A \cap \Delta_{a_{0}}(r), B ; D \cap \Delta_{a_{0}}(r), \widetilde{G}\right)\right)$ which admits the angular limit $f$ on $\left.\mathbb{X}^{\mathrm{o}}\left(A \cap A^{*} \cap \Delta_{a_{0}}(r), B \cap B^{*} ; D \cap \Delta_{a_{0}}(r), \widetilde{G}\right)\right)$. Define

$$
f_{0}:=\left\{\begin{array}{ll}
f & \text { on }\left(A \cap A^{*} \cap \Delta_{a_{0}}(r)\right) \times V \\
\widetilde{f} & \text { on } D_{a_{0}, r, \frac{1}{2}} \times \mathcal{G}
\end{array} .\right.
$$

Then $f_{0} \in \mathcal{O}_{s}(Y),\left.f_{0}\right|_{\left(A \cap A^{*} \cap \Delta_{a_{0}}(r)\right) \times \mathcal{G}}$ is measurable, and

$$
\lim _{\mathcal{A}_{\alpha}(\zeta) \ni z \rightarrow \zeta} f_{0}(z, w)=f(\zeta, w)=f_{0}(\zeta, w),
$$

$$
(\zeta, w) \in\left(A \cap A^{*} \cap \Delta_{a_{0}}(r)\right) \times \mathcal{G}, 0<\alpha<\frac{\pi}{2} .
$$

Consequently, we are able to apply Theorem 3.2 to $f_{0}$ in order to obtain a function $\hat{f}_{0}$ holomorphic on

$$
\widehat{Y}=\left\{(z, w) \in D_{a_{0}, r, \frac{1}{2}} \times V: 2 \omega\left(z, A \cap \Delta_{a_{0}}(r), D \cap \Delta_{a_{0}}(r)\right)+\omega(w, \mathcal{G}, V)<1\right\}
$$

such that $\hat{f}_{0}=\tilde{f}$ on $D_{a_{0}, r, \delta} \times \mathcal{G}$ and

$$
\lim _{\mathcal{A}_{\alpha}(\zeta) \ni z \rightarrow \zeta} \hat{f}_{0}(z, w)=f(\zeta, w)=: \hat{f}_{0}(\zeta, w),(\zeta, w) \in\left(A \cap A^{*} \cap \Delta_{a_{0}}(r)\right) \times V, 0<\alpha<\frac{\pi}{2} .
$$

We have just extended $\hat{f}_{0}$ to $\widehat{Y} \cup\left(\left(A \cap A^{*} \cap \Delta_{a_{0}}(r)\right) \times V\right)$. Fix $s^{-}, s^{+}>0$ such that $\rho^{-}<s^{-}<\rho<s^{+}<\rho^{+}$, and consider the annulus

$$
Q:=\left\{w \in \mathbb{C}: s^{-}<\left|w-w_{0}\right|<s^{+}\right\} .
$$

Let $\delta$ be such that

$$
0<\delta<\frac{1}{2}\left(1-\sup _{w \in Q} \omega(w, \mathcal{G}, V)\right)
$$

Using this and applying Lemma [3.6, we see that $D_{a_{0}, r, \delta} \times \bar{Q} \subset \widehat{Y}$. Therefore, $\hat{f}_{0}$ is holomorphic on $D_{a_{0}, r, \delta} \times Q$ and continuous on $D_{a_{0}, r, \delta} \times \bar{Q}$. Moreover, for any 
$a \in A \cap A^{*} \cap \Delta_{a_{0}}(r)$ the function $\hat{f}_{0}(a, \cdot)$ is holomorphic on $Q$ and continuous on $\bar{Q}$. Therefore, by Cauchy formula we have

$$
\begin{aligned}
\hat{f}_{0}(z, w) & =\frac{1}{2 i \pi} \int_{\left|\eta-w_{0}\right|=s^{+}} \frac{\hat{f}_{0}(z, \eta)}{\eta-w}-\frac{1}{2 i \pi} \int_{\left|\eta-w_{0}\right|=s^{-}} \frac{\hat{f}_{0}(z, \eta)}{\eta-w} \\
& =: \quad \hat{f}^{+}(z, w)+\hat{f}^{-}(z, w), \quad z \in D_{a_{0}, r, \delta} \cup\left(A \cap A^{*} \cap \Delta_{a_{0}}(r)\right), w \in Q .
\end{aligned}
$$

where $\hat{f}^{+} \in \mathcal{O}\left(D_{a_{0}, r, \delta} \times \Delta_{w_{0}}\left(s^{+}\right)\right)$and $\hat{f}^{-} \in \mathcal{O}\left(D_{a_{0}, r, \delta} \times\left(\mathbb{C} \backslash \bar{\Delta}_{w_{0}}\left(s^{-}\right)\right)\right)$.

Recall from (4.4) and the hypotheses that for any $a \in A \cap A^{*} \cap \Delta_{a_{0}}(r)$ the function $\hat{f}_{0}(a, \cdot)$ extends holomorphically to $G \backslash M_{a}$. Consequently, for any $a \in A \cap A^{*} \cap \Delta_{a_{0}}(r)$ the function $\hat{f}^{-}(a, \cdot)$ extends holomorphically to $\mathbb{C} \backslash\left(M_{a} \cap \bar{\Delta}_{w_{0}}\left(s^{-}\right)\right)$. Using (4.4) and the above integral formula for $\hat{f}^{-} \in \mathcal{O}\left(D_{a_{0}, r, \delta} \times Q\right)$, we see that

$\lim _{(z, w) \rightarrow(\zeta, \eta), z \in \mathcal{A}_{\alpha}(\zeta)} \hat{f}^{-}(z, w)=\hat{f}^{-}(\zeta, \eta), \quad(\zeta, \eta) \in\left(A \cap A^{*} \cap \Delta_{a_{0}}(r)\right) \times Q, 0<\alpha<\frac{\pi}{2}$.

Now, we are in the position to apply Theorem 3.8 to $\hat{f}^{-}$. Consequently, there exists a relatively closed pluripolar set $\widetilde{S} \subset D_{a_{0}, r, \delta} \times \mathbb{C}$ such that $\hat{f}^{-}$extends holomorphically to a function $\widetilde{\widetilde{f}}^{-} \in \mathcal{O}\left(\left(D_{a_{0}, r, \delta} \times \mathbb{C}\right) \backslash \widetilde{S}\right)$.

Since $\hat{f}_{0}=\hat{f}^{+}+\hat{f}^{-}$, the function $\hat{f}_{0}$ extends holomorphically to a function (still denoted by $) \hat{f}_{0}:=\hat{f}^{+}+\widetilde{\widetilde{f}}^{-} \in \mathcal{O}\left(\left(D_{a_{0}, r, \delta} \times \Delta_{w_{0}}\left(s^{+}\right)\right) \backslash \widetilde{S}\right)$.

To prove Part 1) and Part 2) fix an arbitrary $a_{1} \in A \cap A^{*} \cap \Delta_{a_{0}}(r)$ and $w_{1} \in \Delta_{w_{0}}(\rho)$. Since $M_{a_{1}}$ is polar in $G$, there exists a smooth curve $\alpha:[0,1] \rightarrow \mathbb{C} \backslash M_{a_{1}}$ such that $\alpha(0) \in \widetilde{G}$ and $\alpha(1)=w_{1}$. Moreover, using (4.1) and the hypothesis that $M$ is a relatively closed subset of $W$, we may find $r_{1}>0$ so small that $\widetilde{V}:=$ $\widetilde{G} \cup\left(\alpha([0,1])+\Delta_{0}\left(r_{1}\right)\right)$ is a Jordan domain and that

$$
\Delta_{a_{1}}\left(r_{1}\right) \Subset \Delta_{a_{0}}(r), \quad\left(\Delta_{a_{1}}\left(r_{1}\right) \times \widetilde{V}\right) \cap M=\varnothing .
$$

Using this, (4.1), and the hypotheses on $f$ in the Main Theorem, we are able to apply Theorem 3.3 to the function $f$ restricted to $\mathbb{X}\left(A \cap \Delta_{a_{1}}\left(r_{1}\right), B ; D \cap \Delta_{a_{1}}\left(r_{1}\right), \widetilde{V}\right)$. Consequently, we obtain $\widehat{f}_{1}=\widehat{f}_{\left(a_{1}, w_{1}\right)} \in \mathcal{O}\left(\widehat{\mathbb{X}}\left(A \cap \Delta_{a_{1}}\left(r_{1}\right), B ; D \cap \Delta_{a_{1}}\left(r_{1}\right), \widetilde{V}\right)\right)$ which admits the angular limit $f$ on $\left.\mathbb{X}^{\circ}\left(A \cap A^{*} \cap \Delta_{a_{1}}\left(r_{1}\right), B \cap B^{*} ; D \cap \Delta_{a_{1}}\left(r_{1}\right), \widetilde{V}\right)\right)$. Fix a $w_{2} \in \mathcal{G}$. Then $w_{2} \in V \cap \widetilde{V}$. Choose $\delta_{1}, \rho_{1}>0$ so small such that

$$
\delta_{1}<1-\omega\left(w_{1}, B, \widetilde{V}\right)
$$

and that

$$
D_{a_{1}, r_{1}, \delta_{1}} \times \Delta_{w_{2}}\left(\rho_{1}\right) \subset\left(D_{a_{0}, r, \delta} \times \Delta_{w_{0}}\left(s^{+}\right)\right) \cap \widehat{\mathbb{X}}\left(A \cap \Delta_{a_{1}}\left(r_{1}\right), B ; D \cap \Delta_{a_{1}}\left(r_{1}\right), \widetilde{V}\right) .
$$

Consequently, using (4.4), we obtain

$$
\begin{aligned}
& \lim _{\mathcal{A}_{\alpha}(\zeta) \ni z \rightarrow \zeta} \hat{f}_{0}(z, w)=\lim _{z \rightarrow \zeta, z \in \mathcal{A}_{\alpha}(\zeta)} \hat{f}_{1}(z, w)=f(\zeta, w), \\
& \zeta \in A \cap A^{*} \cap \Delta_{a_{1}}\left(r_{1}\right), w \in \Delta_{w_{2}}\left(\rho_{1}\right), 0<\alpha<\frac{\pi}{2} .
\end{aligned}
$$


By Theorem 3.9, $\hat{f}_{0}=\hat{f}_{1}$ on $D_{a_{1}, r_{1}, \delta_{1}} \times \Delta_{w_{2}}\left(\rho_{1}\right)$. By shrinking $\rho_{1}$ (if necessary) and by using the fact that $w_{1}, w_{2} \in \widetilde{V}$ and estimate (4.5), we deduce from the latter identity that

$$
\hat{f}_{0}=\hat{f}_{1} \quad \text { on }\left(D_{a_{1}, r_{1}, \delta_{1}} \times \Delta_{w_{1}}\left(\rho_{1}\right)\right) \backslash \widetilde{S}
$$

Now we are in the position to apply Theorem 3.10 to $\hat{f}_{0} \in \mathcal{O}\left(\left(D_{a_{0}, r, \delta} \times \Delta_{w_{0}}\left(s^{+}\right)\right) \backslash \widetilde{S}\right)$ and to the family of functions $\left(\widehat{f}_{\left(a_{1}, w_{1}\right)}\right)$ with $a_{1} \in A \cap A^{*} \cap \Delta_{a_{0}}(r)$ and $w_{1} \in$ $\Delta_{w_{0}}(\rho) \backslash M_{a_{1}}$. Consequently, we obtain the relatively closed pluripolar subset $S \subset$ $D_{a_{0}, r, \delta} \times \Delta_{w_{0}}(\rho)$ satisfying Part 1) and the function $\hat{f} \in \mathcal{O}\left(\left(D_{a_{0}, r, \delta} \times \Delta_{w_{0}}(\rho)\right) \backslash S\right)$. Part 2) follows from (4.6).

The role of strong end-points is illustrated by the following uniqueness theorem.

Theorem 4.2. Let $f \in \mathcal{O}(\Omega)$, where $\Omega$ is a subdomain of $\widehat{W}$. Suppose that there exist $a_{0} \in A \cap A^{*}, r>0$ and an open subset $V \subset G$ such that $\left(A \cap A^{*} \cap \Delta_{a_{0}}(r)\right) \times V$ are contained in the set of strong end-points of $\Omega$ and that the angular limit of $f$ at all points of $\left(A \cap A^{*} \cap \Delta_{a_{0}}(r)\right) \times V$ equals 0 . Then $f \equiv 0$.

Proof. Applying Theorem 3.9 to $f$ restricted to an open set of the form $D_{a_{0}, r_{0}, \delta} \times U \subset$ $\Omega$ for suitable $r_{0}, \delta>0$ and $U \subset G$, the theorem follows.

\section{Proof of the Main Theorem}

We keep the notation in the previous section. Moreover, we introduce some new notation. For any $\zeta \in A, r, R \in(0,1)$ with $\Delta_{0}(R) \cap \widetilde{G} \neq \varnothing$, let

$$
W_{\zeta, r, R}:=\mathbb{X}\left(A \cap \Delta_{\zeta}(r), B ; D \cap \Delta_{\zeta}(r), \Delta_{0}(R) \cup \widetilde{G}\right) .
$$

Similarly, for any $\eta \in B, r, R \in(0,1)$ with $\Delta_{0}(R) \cap \widetilde{D} \neq \varnothing$, put

$$
W_{\eta, r, R}:=\mathbb{X}\left(A, B \cap \Delta_{\eta}(r) ; \Delta_{0}(R) \cup \widetilde{D}, G \cap \Delta_{\eta}(r)\right) .
$$

For any $R \in(0,1)$ with $\Delta_{0}(R) \cap \widetilde{D} \neq \varnothing$ and $\Delta_{0}(R) \cap \widetilde{G} \neq \varnothing$, put

$$
W_{R}:=\mathbb{X}\left(A, B ; \Delta_{0}(R) \cup \widetilde{D}, \Delta_{0}\left(R^{\prime}\right) \cup \widetilde{G}\right) .
$$

Fix a sequence $\left(\delta_{n}:=\frac{1}{2^{n}}\right)_{n=1}^{\infty}$.

The proof is divided into several steps. In the first three steps $A$ and $B$ are supposed to be compact.

Step 1. For any $\zeta \in A \cap A^{*}$ and $R \in(0,1)$ with $\Delta_{0}(R) \cap \widetilde{G} \neq \varnothing$, there exists $r \in(0,1)$ and a relatively closed pluripolar subset $\widehat{S}$ of $\widehat{W}_{\zeta, r, R}$ and a function $\hat{f} \in$ $\mathcal{O}\left(\widehat{W}_{\zeta, r, R} \backslash \widehat{S}\right)$ with the following properties:

- $\left(W_{\zeta, r, R}^{o} \cap W_{\zeta, r, R}^{*}\right) \backslash M$ is contained in the set of strong end-points of $\widehat{W}_{\zeta, r, R} \backslash \widehat{S}$.

- $\hat{f}$ admits the angular limit $f$ at all points of $\left(W_{\zeta, r, R}^{o} \cap W_{\zeta, r, R}^{*}\right) \backslash M$. 
Applying Proposition 4.1 to the points $\zeta \in A \cap A^{*}$ and $w \in \bar{\Delta}_{0}(R)$ and using the compactness of $\bar{\Delta}_{0}(R)$, we find $r, \delta \in(0,1), p \in \mathbb{N}$, and for any $j \in\{1, \ldots, p\}$, a point $w_{j} \in \bar{\Delta}_{0}(R)$, a number $\rho_{j}>0$, a relatively closed subset $S_{j} \subset D_{\zeta, r, \delta} \times \Delta_{w_{j}}\left(\rho_{j}\right)$, and a function $\hat{f}_{j} \in \mathcal{O}\left(\left(D_{\zeta, r, \delta} \times \Delta_{w_{j}}\left(\rho_{j}\right)\right) \backslash S_{j}\right)$ such that

- $\bar{\Delta}_{0}(R) \subset \bigcup_{k=1}^{p} \Delta_{w_{k}}\left(\rho_{k}\right)$;

- $\hat{f}_{j}$ admits the angular limit $f$ at all points of $\left(\left(A \cap A^{*} \cap \Delta_{\zeta}(r)\right) \times \Delta_{w_{j}}\left(\rho_{j}\right)\right) \backslash M$.

Using this we are able to apply Theorem 4.2. Consequently,

$$
\hat{f}_{i}=\hat{f}_{j} \quad \text { on }\left(D_{\zeta, r, \delta} \times\left(\Delta_{w_{i}}\left(\rho_{i}\right) \cap \Delta_{w_{j}}\left(\rho_{j}\right) \cap \Delta_{0}(R)\right)\right) \backslash\left(S_{i} \cup S_{j}\right) .
$$

Therefore, we obtain an $\tilde{f} \in \mathcal{O}\left(\left(D_{\zeta, r, \delta} \times \Delta_{0}(R)\right) \backslash S^{\prime}\right)$, where $\tilde{f}=\hat{f}_{j}$ on $\left(D_{\zeta, r, \delta} \times\right.$ $\left.\Delta_{w_{j}}\left(\rho_{j}\right)\right) \backslash S^{\prime}$ and $S^{\prime}:=\bigcup_{j=1}^{p} S_{j}$ is relatively closed pluripolar set. Moreover, $((A \cap$ $\left.\left.A^{*} \cap \Delta_{\zeta}(r)\right) \times \Delta_{0}(R)\right) \backslash M$ is contained in the set of strong end-points of $\left(D_{\zeta, r, \delta} \times\right.$ $\left.\Delta_{0}(R)\right) \backslash S^{\prime}$ and $\tilde{f}$ admits the angular limit $f$ at all points of the former set.

On the other hand, applying Theorem 3.3 to the function $f$ restricted to $\mathbb{X}(A \cap$ $\left.\Delta_{\zeta}(r), B ; D \cap \Delta_{\zeta}(r), \widetilde{G}\right)$, we obtain $\widetilde{\widetilde{f}} \in \mathcal{O}\left(\widehat{\mathbb{X}}\left(A \cap \Delta_{\zeta}(r), B ; D \cap \Delta_{\zeta}(r), \widetilde{G}\right)\right)$ which admits the angular limit $f$ on $\left.\mathbb{X}^{\circ}\left(A \cap A^{*} \cap \Delta_{\zeta}(r), B \cap B^{*} ; D \cap \Delta_{\zeta}(r), \widetilde{G}\right)\right)$.

Next, we fix an $n_{0}$ such that $\delta_{n_{0}}<\delta$. For $s \in(0,1)$ let $\widetilde{G}_{s}:=\{w \in \widetilde{G}$ : $\omega(w, B, \widetilde{G})<s\}$. For all $n \geq n_{0}$ let

$$
W_{n}:=\mathbb{X}\left(D_{\zeta, r, \delta_{n}}, \widetilde{G}_{\delta_{n}} ; D_{\zeta, r, 1-\delta_{n}}, \Delta_{0}(R) \cup \widetilde{G}_{1-\delta_{n}}\right) .
$$

Define $f_{n}: W_{n} \backslash S^{\prime} \rightarrow \mathbb{C}$ as follows

$$
f_{n}:=\left\{\begin{array}{ll}
\tilde{f}, & \text { on }\left(D_{\zeta, r, \delta_{n}} \times\left(\Delta_{0}(R) \cup \widetilde{G}_{1-\delta_{n}}\right)\right) \backslash S^{\prime} \\
\widetilde{f}, & \text { on } D_{\zeta, r, 1-\delta_{n}} \times \widetilde{G}_{\delta_{n}}
\end{array} ;\right.
$$

here we have applied Theorem 3.9 in order to show that $\tilde{f}=\tilde{\widetilde{f}}$ on the overlapping set. Clearly, $f_{n} \in \mathcal{O}\left(W_{n} \backslash S^{\prime}\right)$. Therefore, applying Theorem 3.1 and Theorem 3.4 to $W_{n} \backslash S^{\prime}$, we obtain a relatively closed pluripolar subset $\widehat{S}_{n}$ of $\widehat{W}_{n}$ with $\widehat{S}_{n} \cap W_{n} \subset S^{\prime}$ and a function $\hat{f}_{n} \in \mathcal{O}\left(\widehat{W}_{n} \backslash \widehat{S}_{n}\right)$ with $\hat{f}_{n}=f_{n}$ on $W_{n} \backslash S^{\prime}$. Now, using Lemma 3.6, we define

$$
\begin{aligned}
X_{n}:=\quad \mathbb{X}\left(A \cap A^{*} \cap \Delta_{\zeta}(r), B \cap B^{*} ; D_{\zeta, r, 1-\delta_{n}}, \Delta_{0}(R) \cup \widetilde{G}_{1-\delta_{n}}\right), \\
\widehat{X}_{n}:=\quad\left\{(z, w) \in D_{\zeta, r, 1-\delta_{n}} \times\left(\Delta_{0}(R) \cup \widetilde{G}_{1-\delta_{n}}\right): \widetilde{\omega}\left(z, A \cap A^{*} \cap \Delta_{\zeta}(r), D_{\zeta, r, 1-\delta_{n}}\right)\right. \\
\left.\quad+\widetilde{\omega}\left(w, B \cap B^{*}, \Delta_{0}(R) \cup \widetilde{G}_{1-\delta_{n}}\right)<1\right\} .
\end{aligned}
$$

Then it follows from (5.1) that $\hat{f}_{n}$ restricted to $\widehat{X}_{n} \backslash \widehat{S}_{n}$, admits the angular limit $f$ at all points of $X_{n}^{\mathrm{o}}$ and the latter set is contained in the set of strong end-points of $\widehat{X}_{n} \backslash \widehat{S}_{n}$. Therefore, applying Theorem 4.2 we see that $\hat{f}_{n}=\hat{f}_{n+1}$ on $\widehat{X}_{n} \backslash\left(\widehat{S}_{n} \cup \widehat{S}_{n+1}\right)$. 
Moreover, using Theorem 3.10 we may assume that $\widehat{S}_{n+1} \cap \widehat{X}_{n} \subset \widehat{S}_{n}$. Next, we will show that $\widehat{X}_{n} \nearrow \widehat{W}_{\zeta, r, R}$ as $n \nearrow \infty$. To see this it suffices to observe by Lemma 3.6 that

$$
\begin{aligned}
\omega\left(\cdot, A \cap A^{*} \cap \Delta_{\zeta}(r), D_{\zeta, r, 1-\delta_{n}}\right) & \searrow \omega\left(\cdot, A \cap A^{*}, D \cap \Delta_{\zeta}(r)\right), \\
\omega\left(\cdot, B \cap B^{*}, \Delta_{0}(R) \cup \widetilde{G}_{1-\delta_{n}}\right) & \searrow \omega\left(\cdot, B \cap B^{*}, \widetilde{G}\right),
\end{aligned}
$$

when $n \nearrow \infty$. Now we are in the position to apply Theorem 3.11 to the functions $\hat{f}_{n} \in \mathcal{O}\left(\widehat{X}_{n} \backslash \widehat{S}_{n}\right)$ for $n \geq n_{0}$. Consequently, we obtain the desired relatively closed pluripolar subset $\widehat{S}$ of $\widehat{W}_{\zeta, r, R}$ and the desired extension function $\hat{f}$. This finishes Step 1.

Step 2. For any $R \in(0,1)$ such that $\Delta_{0}(R) \cup \widetilde{D}$ and $\Delta_{0}(R) \cup \widetilde{G}$ are Jordan domains, there exist a relatively closed pluripolar subset $\widehat{S}$ of $\widehat{W}_{R}$ and a function $\hat{f} \in \mathcal{O}\left(\widehat{W}_{R} \backslash \widehat{S}\right)$ such that the set $\left(W_{R}^{o} \cap W_{R}^{*}\right) \backslash M$ is contained in the set of strong end-points of $\widehat{W}_{R} \backslash \widehat{S}$ and that $\hat{f}$ admits the angular limit $f$ at all points of the former set.

Choose a sequence of closed subsets $\left(\widetilde{A}_{m}\right)_{m=1}^{\infty}\left(\operatorname{resp} .\left(\widetilde{B}_{m}\right)_{m=1}^{\infty}\right)$ of $\partial D(\operatorname{resp} . \partial G)$ such that

$$
\begin{aligned}
& \operatorname{mes}\left(\widetilde{A}_{m}\right)>0, \widetilde{A}_{m} \subset \widetilde{A}_{m+1} \subset A \cap A^{*}, \operatorname{mes}\left(A \backslash \bigcup_{m=1}^{\infty} \widetilde{A}_{m}\right)=0, \\
& \operatorname{mes}\left(\widetilde{B}_{m}\right)>0, \widetilde{B}_{m} \subset \widetilde{B}_{m+1} \subset B \cap B^{*}, \operatorname{mes}\left(B \backslash \bigcup_{m=1}^{\infty} \widetilde{B}_{m}\right)=0 .
\end{aligned}
$$

Let

$\mathcal{W}_{m}:=\mathbb{X}\left(\widetilde{A}_{m}, \widetilde{B}_{m} ; \Delta_{0}(R) \cup \widetilde{D}, \Delta_{0}(R) \cup \widetilde{G}\right), \quad \widehat{\mathcal{W}}_{m}:=\widehat{\mathbb{X}}\left(\widetilde{A}_{m}, \widetilde{B}_{m} ; \Delta_{0}(R) \cup \widetilde{D}, \Delta_{0}(R) \cup \widetilde{G}\right)$.

First, we will show that for every $m$ there exist a relatively closed pluripolar subset $\widehat{\mathcal{S}}_{m}$ of $\widehat{\mathcal{W}}_{m}$ and a function $\widetilde{f}_{m} \in \mathcal{O}\left(\widehat{\mathcal{W}}_{m} \backslash \widehat{\mathcal{S}}\right)$ such that the set $\left(\mathcal{W}_{m}^{\circ} \cap \mathcal{W}_{m}^{*}\right) \backslash M$ is contained in the set of strong end-points of $\widehat{\mathcal{W}}_{m} \backslash \widehat{\mathcal{S}}_{m}$ and that $\widetilde{f}_{m}$ admits the angular limit $f$ at all points of the former set. For this purpose fix an $m \in \mathbb{N}$.

Applying Step 1 and using a compactness argument with respect to $\widetilde{A}_{m}$ we may find $K$ points $\zeta_{1}, \ldots, \zeta_{K} \in A \cap A^{*}$ and $K$ numbers $r_{1}, \ldots, r_{K}>0$ with the following properties:

- $\widetilde{A}_{m} \subset \bigcup_{k=1}^{K} \Delta_{\zeta_{k}}\left(r_{k}\right)$ and $D \cap \bigcup_{k=1}^{K} \Delta_{\zeta_{k}}\left(r_{k}\right) \subset \widetilde{D}$

- for every $1 \leq k \leq K$, there are a relatively closed pluripolar subset $S_{k}$ of $\widehat{W}_{\zeta_{k}, r_{k}, R}$ and a function $\hat{g}_{k} \in \mathcal{O}\left(\widehat{W}_{\zeta_{k}, r_{k}, R} \backslash S_{k}\right)$ such that the set $\left(W_{\zeta_{k}, r_{k}, R}^{\mathrm{o}} \cap\right.$ $\left.W_{\zeta_{k}, r_{k}, R}^{*}\right) \backslash M$ is contained in the set of strong end-points of $\widehat{W}_{\zeta_{k}, r_{k}, R} \backslash S_{k}$ and that $\hat{g}_{k}$ admits the angular limit $f$ at all points of the former set.

Similarly, using Step 1 again but exchanging the role between $A$ and $B$ (resp. $D$ and $G$ ), we may find $L$ points $\eta_{1}, \ldots, \eta_{L} \in B \cap B^{*}$ and $L$ numbers $s_{1}, \ldots, s_{L}>0$ with the following properties: 
- $\widetilde{B}_{m} \subset \bigcup_{l=1}^{L} \Delta_{\eta_{l}}\left(s_{l}\right)$ and $G \cap \bigcup_{l=1}^{L} \Delta_{\eta_{l}}\left(s_{l}\right) \subset \widetilde{G}$;

- for every $1 \leq l \leq L$, there are a relatively closed pluripolar subset $T_{l}$ of $\widehat{W}_{\eta_{l}, s_{l}, R}$ and a function $\hat{h}_{l} \in \mathcal{O}\left(\widehat{W}_{\eta_{l}, s_{l}, R} \backslash T_{l}\right)$ such that the set $\left(W_{\eta_{l}, s_{l}, R}^{\mathrm{o}} \cap\right.$ $\left.W_{\eta_{l}, s_{l}, R}^{*}\right) \backslash M$ is contained in the set of strong end-points of $\widehat{W}_{\eta_{l}, s_{l}, R} \backslash T_{l}$ and that $h_{l}$ admits the angular limit $f$ at all points of the former set.

Put $S:=\bigcup_{k=1}^{K} S_{k}$ and $T:=\bigcup_{l=1}^{L} T_{l}$. For every $n \geq 1$ let

$$
\begin{aligned}
A_{n} & :=\bigcup_{k=1}^{K} D_{\zeta_{k}, r_{k}, \delta_{n}}, \quad B_{n}:=\bigcup_{l=1}^{L} G_{\eta_{l}, s_{l}, \delta_{n}}, \\
D_{n} & :=\left\{z \in \Delta_{0}(R) \cup \widetilde{D}: \omega\left(z, \widetilde{A}_{m}, \Delta_{0}(R) \cup \widetilde{D}\right)<1-\delta_{n}\right\}, \\
G_{n} & :=\left\{w \in \Delta_{0}(R) \cup \widetilde{G}: \omega\left(w, \widetilde{B}_{m}, \Delta_{0}(R) \cup \widetilde{G}\right)<1-\delta_{n}\right\}, \\
W_{n} & :=\mathbb{X}\left(A_{n}, B_{n} ; D_{n}, G_{n}\right), \quad X_{n}:=\mathbb{X}\left(\widetilde{A}_{m} \cap \widetilde{A}_{m}^{*}, \widetilde{B}_{m} \cap \widetilde{B}_{m}^{*} ; D_{n}, G_{n}\right), \\
\widehat{\widetilde{X}}_{n} & :=\left\{(z, w) \in D_{n} \times G_{n}: \widetilde{\omega}\left(z, \widetilde{A}_{m} \cap \widetilde{A}_{m}^{*}, D_{n}\right)+\widetilde{\omega}\left(w, \widetilde{B}_{m} \cap \widetilde{B}_{m}^{*}, G_{n}\right)<1\right\},
\end{aligned}
$$

where in the last line we can apply Lemma 3.6 since $\Delta_{0}(R) \cup \widetilde{D}$ and $\Delta_{0}(R) \cup \widetilde{G}$ are Jordan domains. Applying Theorem 4.2 and Theorem 3.11 , we may glue $\left(\hat{g}_{k}\right)_{k=1}^{K}$ together in order to define the function $g_{n}:\left(A_{n} \times G_{n}\right) \backslash S \longrightarrow \mathbb{C}$ as follows

$$
g_{n}:=\hat{g}_{k} \quad \text { on }\left(D_{\zeta_{k}, r_{k}, \delta_{n}} \times G_{n}\right) \backslash S .
$$

Similarly, we may glue $\left(\hat{h}_{l}\right)_{l=1}^{L}$ together in order to define the function $h_{n}:\left(D_{n} \times\right.$ $\left.B_{n}\right) \backslash T \longrightarrow \mathbb{C}$ as follows

$$
h_{n}:=\hat{h}_{l} \quad \text { on }\left(D_{n} \times G_{\eta_{l}, s_{l}, \delta_{n}}\right) \backslash T .
$$

Finally, we glue $g_{n}$ and $h_{n}$ together in order to define the function $f_{n}: W_{n} \backslash(T \cup$ $S) \longrightarrow \mathbb{C}$ as follows

$$
f_{n}:=\left\{\begin{array}{ll}
g_{n} & \text { on }\left(A_{n} \times G_{n}\right) \backslash S \\
h_{n}, & \text { on }\left(D_{n} \times B_{n}\right) \backslash T
\end{array} .\right.
$$

The remaining part of the proof follows along the same lines as in Step 1. Applying Theorem 3.1 and Theorem 3.4 to $W_{n} \backslash(S \cup T)$, we obtain a relatively closed pluripolar subset $\widehat{S}_{n}$ of $\widehat{W}_{n}$ with $\widehat{S}_{n} \cap W_{n} \subset(S \cup T)$ and a function $\hat{f}_{n} \in \mathcal{O}\left(\widehat{W}_{n} \backslash \widehat{S}_{n}\right)$ with $\hat{f}_{n}=f_{n}$ on $W_{n} \backslash(S \cup T)$. In particular, it follows from (5.4) that $\hat{f}_{n}$ restricted to $\widehat{\widetilde{X}}_{n} \backslash \widehat{S}_{n}$, admits the angular limit $f$ at all points of $X_{n}^{\mathrm{o}} \backslash M$. Here observe that the latter set is contained in the set of strong end-points of $\widehat{\widetilde{X}}_{n} \backslash \widehat{S}_{n}$. Therefore, applying Theorem 4.2 we see that $\hat{f}_{n}=\hat{f}_{n+1}$ on $\widehat{\widetilde{X}}_{n} \backslash\left(\widehat{S}_{n} \cup \widehat{S}_{n+1}\right)$. Moreover, using Theorem 3.10 we may assume that $\widehat{S}_{n+1} \cap \widehat{\widetilde{X}}_{n} \subset \widehat{S}_{n}$. Next, an application of Lemma 3.6 gives 
that $\widehat{\widetilde{X}}_{n} \nearrow \widehat{\mathcal{W}}_{m}$ as $n \nearrow \infty$. Now we are in the position to apply Theorem 3.11 to the functions $\hat{f}_{n} \in \mathcal{O}\left(\widehat{\widetilde{X}}_{n} \backslash \widehat{S}_{n}\right)$ for $n \geq 2$. Consequently, we obtain the desired relatively closed pluripolar subset $\widehat{\mathcal{S}}_{m}$ of $\widehat{\mathcal{W}}_{m}$ and the desired extension function $\widetilde{f}_{m}$.

Using (15.2) -(5.3) and (2.1) we see that $\widehat{\mathcal{W}}_{m} \nearrow \widehat{W}_{R}$ as $m \nearrow \infty$. Therefore, applying Theorem 4.2, Theorem 3.10 and Theorem 3.11, Step 2 follows.

Step 3. Completion of the case where $A$ and $B$ are compact.

Fix $R \in(0,1)$ such that $\Delta_{0}(R) \cup \widetilde{D} \neq \varnothing$ and $\Delta_{0}(R) \cup \widetilde{G} \neq \varnothing$ are Jordan domains. Choose a sequence $\left(R_{n}\right)_{n=1}^{\infty}$ such that $R_{n}>R, R_{n} \nearrow 1$ as $n \nearrow \infty$. For $n \geq 1$ put $W_{n}:=\mathbb{X}\left(A, B ; \Delta_{0}\left(R_{n}\right) \cup \widetilde{D}, \Delta_{0}\left(R_{n}\right) \cup \widetilde{G}\right)$. Applying the result of Step 2, we may find, for every $n \geq 1$, a relatively closed pluripolar subset $\widehat{M}_{n}$ of $\widehat{W}_{n}$ and a function $\hat{f}_{n} \in \mathcal{O}\left(\widehat{W}_{n} \backslash \widehat{M}_{n}\right)$ such that $\left(W_{n}^{*} \cap W_{n}^{\mathrm{o}}\right) \backslash M$ is contained in the set of strong end-points of $\widehat{W}_{n} \backslash \widehat{M}_{n}$ and that $\hat{f}_{n}$ admits the angular limit $f$ at all points of the former set. Since $\widehat{W}_{n} \nearrow \widehat{W}$ as $n \nearrow \infty$, we conclude this step by applying Theorem 4.2. Theorem 3.10 and Theorem 3.11 as in Step 2.

Step 4. The general case.

Choose a sequence of closed subsets $\left(A_{n}\right)_{n=1}^{\infty}\left(\operatorname{resp} .\left(B_{n}\right)_{n=1}^{\infty}\right)$ of $\partial D(\operatorname{resp} . \partial G)$ such that

$$
\begin{aligned}
& \operatorname{mes}\left(A_{n}\right)>0, A_{n} \subset A_{n+1} \subset A, \operatorname{mes}\left(A \backslash \bigcup_{n=1}^{\infty} A_{n}\right)=0, \\
& \operatorname{mes}\left(B_{n}\right)>0, B_{n} \subset B_{n+1} \subset B, \operatorname{mes}\left(B \backslash \bigcup_{n=1}^{\infty} B_{n}\right)=0 .
\end{aligned}
$$

Let $W_{n}:=\mathbb{X}\left(A_{n}, B_{n} ; D, G\right)$. Applying the hypotheses to $\left.f\right|_{W_{n} \backslash M}$ for $n \geq 1$, we obtain a relatively closed pluripolar subset $\widehat{M}_{n}$ of $\widehat{W}_{n}$ and a function $\hat{f}_{n} \in \mathcal{O}\left(\widehat{W}_{n} \backslash \widehat{M}_{n}\right)$ such that $\left(W_{n}^{*} \cap W_{n}^{\mathrm{o}}\right) \backslash M$ is contained in the set of strong end-points of $\widehat{W}_{n} \backslash \widehat{M}_{n}$ and that $\hat{f}_{n}$ admits the angular limit $f$ at all points of the former set. Using (2.1) we see that $\widehat{W}_{n} \nearrow \widehat{W}$ as $n \nearrow \infty$. Therefore, arguing as at the end of the previous step, Step 4 follows.

\section{REFERENCES}

[1] R. A. Airapetyan, G. M. Henkin, Analytic continuation of CR-functions across the "edge of the wedge", Dokl. Akad. Nauk SSSR, 259 (1981), 777-781 (Russian). English transl.: Soviet Math. Dokl., 24 (1981), 128-132.

[2] R. A. Airapetyan, G. M. Henkin, Integral representations of differential forms on CauchyRiemann manifolds and the theory of CR-functions. II, Mat. Sb., 127(169), (1985), 92-112, (Russian). English transl.: Math. USSR-Sb. 55 (1986), 99-111.

[3] D. H. Armitage, S. J. Gardiner, Classical Potential Theory, Springer Monographs in Mathematics, Springer-Verlag London, Ltd., London, 2001. xvi+333 pp.

[4] E M. Chirka, The extension of pluripolar singularity sets, Proc. Steklov Inst. Math. 200 (1993), 369-373.

[5] L. M. Drużkowski, A generalization of the Malgrange-Zerner theorem, Ann. Polon. Math., 38, (1980), 181-186. 
[6] A. A. Gonchar, On analytic continuation from the "edge of the wedge" theorem, Ann. Acad. Sci. Fenn. Ser. A.I: Mathematica, 10, (1985), 221-225.

[7] A. A. Gonchar, On Bogolyubov's "edge-of-the-wedge" theorem, Tr. Mat. Inst. Steklova 228 (2000), Probl. Sovrem. Mat. Fiz. 24-31 (Russian). English transl.: Proc. Steklov Inst. Math., 228, (2000), 18-24.

[8] S. A. Imomkulov, J. U. Khujamov, On holomorphic continuation of functions along boundary sections, Math. Bohem., 130 (2005), no. 3, 309-322.

[9] S. A. Imomkulov, On the holomorphic continuation of functions defined on a boundary pencil of complex lines, (Russian) Izv. Ross. Akad. Nauk Ser. Mat., 69 (2005), no. 2, 125-144; translation in Izv. Math. 69 (2005), no. 2, 345-363.

[10] M. Jarnicki, P. Pflug, Extension of Holomorphic Functions, de Gruyter Expositions in Mathematics 34, Walter de Gruyter, 2000.

[11] M. Jarnicki, P. Pflug, An extension theorem for separately holomorphic functions with analytic singularities, Ann. Pol. Math., 80, (2003), 143-161.

[12] M. Jarnicki, P. Pflug, An extension theorem for separately holomorphic functions with pluripolar singularities, Trans. Amer. Math. Soc., 355, No. 3, (2003), 1251-1267.

[13] M. Jarnicki, P. Pflug, An extension theorem for separately meromorphic functions with pluripolar singularities, Kyushu J. Math., 57, (2003), No. 2, 291-302.

[14] M. Jarnicki, P. Pflug, A general cross theorem with singularities, preprint (2006), 27 pages.

[15] H. Komatsu, A local version of Bochner's tube theorem, J. Fac. Sci., Univ. Tokyo, Sect. I A 19, (1972), 201-214.

[16] V.-A. Nguyên, A general version of the Hartogs extension theorem for separately holomorphic mappings between complex analytic spaces, Ann. Scuola Norm. Sup. Pisa Cl. Sci., (2005), serie V, Vol. IV(2), 219-254.

[17] V.-A. Nguyên, A unified approach to the theory of separately holomorphic mappings, preprint of the ICTP, Trieste-Italy (2007), arXiv:0704.0897v1, 53 pages,

[18] P. Pflug and V.-A. Nguyên, A boundary cross theorem for separately holomorphic functions, Ann. Polon. Math., 84, (2004), no. 3, 237-271.

[19] P. Pflug and V.-A. Nguyên, Boundary cross theorem in dimension 1, Ann. Polon. Math., 90(2), (2007), 149-192.

[20] P. Pflug and V.-A. Nguyên, Generalization of a theorem of Gonchar, Ark. Mat., 45, (2007), $105-122$.

[21] P. Pflug and V.-A. Nguyên, Envelope of holomorphy for boundary cross sets, Arch. Math. (Basel), to appear, 12 pages.

[22] P. Pflug and V.-A. Nguyên, Cross theorems with singularities, in preparation, 22 pages.

[23] M. Zerner, Quelques résultats sur le prolongement analytique des fonctions de variables complexes, Séminaire de Physique Mathématique, unpublished manuscript.

Peter Pflug, Carl von Ossietzky Universität Oldenburg, Fachbereich Mathematik, Postfach 2503, D-26111, Oldenburg, Germany

E-mail address: pflug@mathematik.uni-oldenburg.de

Viêt-Anh Nguyên, Mathematics Section, The Abdus Salam international centre FOR TheOREtical PHYSics, Strada COSTIERA, 11, 34014 Trieste, ItAly

E-mail address: vnguyen0@ictp.trieste.it 